\title{
The MD4 Message Digest Algorithm
}

\author{
Ronald L. Rivest* \\ Laboratory for Computer Science \\ Massachusetts Institute of Technology \\ Cambridge, MA 02139
}

\begin{abstract}
The MD4 message digest algorithm takes an input message of arbitrary length and produces an output 128-bit "fingerprint" or "message digest", in such a way that it is (hopefully) computationally infeasible to produce two messages having the same message digest, or to produce any message having a given prespecified target message digest. The MD4 algorithm is thus ideal for digital signature applications: a large file can be securely "compressed" with MD4 before being signed with (say) the RSA public-key cryptosystem.

The MD4 algorithm is designed to be quite fast on 32-bit machines. For example, on a SUN Sparc station, MD4 runs at $1,450,000$ bytes/second $(11.6$ $\mathrm{Mbit} / \mathrm{sec}$ ). In addition, the MD4 algorithm does not require any large substitution tables; the algorithm can be coded quite compactly.

The MD4 algorithm is being placed in the public domain for review and possible adoption as a standard.
\end{abstract}

\section{Introduction}

One-way functions were first described in the mid-1970's, as a means to avoid having to store passwords in a time-shared computer system. (This idea is due to Needham (see Wilkes [10, page 91]) and Evans, Kantrowitz, and Weiss [4].)

In 1976, Diffie and Hellman [3] began the exploration of the relationship between one-way functions and other kinds of cryptographic operations, a theme that continues to the present day. They also propose the operation of exponentiating modulo a prime as a candidate one-way function, attributing this idea to John Gill.

A major application of one-way functions was given by Davies and Price in 1980 [2]. They introduce the idea of signing a message $M$ by signing $h(M)$ with a publickey cryptosystem, where $h$ is a one-way function. This procedure has the advantage of permitting considerable improvement in efficiency if $M$ is long, since computing

*Supported by RSA Data Security (Redwood City, California, 94065). email address: rivestotheory. Ics.mit. edu 
$h(M)$ and signing the result can take much less time than directly signing all of $M$ note that $h$ can be fast to compute and $h(M)$ can be short (say 128 bits). The value $h(M)$ is often called the "message digest" of the message $M$. This application is the motivation for the development of the MD4 algorithm.

The theoretical importance of one-way functions as a foundation for cryptography is becoming increasingly clear. Although there has been much work in this area, I'll cite only a couple of references, since the present paper is oriented more towards the practical utilization of one-way functions than towards their theoretical importance. First, Impagliazzo, Levin, and Luby [5] have recently shown that the existence of oneway functions is necessary and sufficient for the existence of secure pseudo-random generators. Using known results, this implies that secure private-key encryption and secure zero-knowledge protocols can be based on any one-way function. Second, Rompel [9] has recently shown (building on the work of Naor and Yung [8]) that the existence of one-way functions is necessary and sufficient for the existence of secure digital signature schemes.

Turning to the practical utilization of one-way functions, it is clear that high speed is a major design criterion. It is desirable to be able to sign very large files (e.g., megabytes in length) very quickly, and to verify these signatures very quickly as well. In one application, for example, it is desired to check the signature on an executable code module before loading and executing it, in order to verify the authenticity of the code module.

There have been many concrete proposals for efficient one-way functions; I don't attempt to survey these proposals here, but merely to give a couple of examples. One approach is to base the one-way function on an efficient conventional encryption scheme. For example, Merkle [7] shows how to construct a secure one-way function if DES is a good random block cipher. However, even his fastest method only hashes 18 bits of message per application of DES. As a second example, Damgård [1] studies the design of one-way functions, proves some theorems regarding the security of oneway functions that work in a "block by block" manner, and proposes a fast one-way function based on the knapsack problem. The design of MD4 was influenced by Damgård's work.

\section{Overview and Design Goals}

The first design goal is, of course, security: it should be computationally infeasible to find two messages $M_{1}$ and $M_{2}$ that have the same message digest. Here we define a task to be "computationally infeasible" if it requires more than $2^{64}$ operations.

The goal of security is to be achieved directly, without assumptions. That is, we do not wish to design a hash function that is secure if, say, factoring is difficult. While much of cryptography is based on such reductions, "the buck stops" at oneway functions-these need to be designed to be difficult to invert without any further assumptions.

The second design goal is speed: the algoritim should be as fast as possible. Here 
we are interested in speed in software; designs that require special-purpose hardware are of limited interest. In particular, we are interested in algorithms that are fast on 32-bit architectures, since that is becoming the dominant standard processor architecture. Thus, the algorithm should be based on a simple set of primitive operations on 32 -bit words.

The third design goal is simplicity and compactness: the algorithm should be simple to describe and simple to program, without requiring large programs or substitution tables. This is not only desirable from an engineering standpoint, but also desirable from a security viewpoint, since a simple algorithm is more likely to receive the necessary critical review.

A fourth design goal was to favor little-endian architectures. Some processor architectures (such as the Intel $80 \times x x$ line) are "little endian": they store the leastsignificant byte of a word in the low-address byte position. Others (such as a SUN Sparcstation) are "big-endian": the most-significant byte of a word goes in the lowaddress byte position. This distinction is significant when treating a message as a sequence of 32-bit words, since one architecture or the other will have to byte-reverse each word before processing. Since the big-endian processors are generally faster (it seems), it was decided to let them do the reversing. This incurs a performance penalty of about $25 \%$.

\section{Terminology and Notation}

In this note a word is a 32 -bit quantity and a byte is an 8-bit quantity. A sequence of bits can be interpreted in a natural manner as a sequence of bytes, where each consecutive group of 8 bits is interpreted as a byte with the high-order (most significant) bit of each byte listed first. Similarly, a sequence of bytes can be interpreted as a sequence of 32-bit words, where each consecutive group of 4 bytes is interpreted as a word with the low-order (least significant) byte given first.

Let the symbol "+" denote addition of words (i.e., modulo- $2^{32}$ addition). Let ( $X<<<s$ ) denote the 32 -bit value obtained by circularly shifting (rotating) $X$ left by $s$ bit positions. Let $\neg X$ denote the bit-wise complement of $X$, and let $X \vee Y$ denote the bit-wise OR of $X$ and $Y$. Let $X \oplus Y$ denote the bit-wise XOR of $X$ and $Y$, and let $X Y$ denote the bit-wise AND of $X$ and $Y$.

\section{MD4 Algorithm Description}

We begin by supposing that we have a $b$-bit message as input, and that we wish to find its message digest. Here $b$ is an arbitrary nonnegative integer; $b$ may be zero, it need not be a multiple of 8 , and it may be arbitrarily large. We imagine the bits of the message written down as follows:

$$
m_{0} m_{1} \ldots m_{b-1}
$$

The following five steps are performed to compute the message digest of the message. 


\section{Step 1. Append padding bits}

The message is padded (extended) so that its length (in bits) is congruent to 448, modulo 512. That is, the message is extended so that it is just 64 bits shy of being a multiple of 512 bits long. Padding is always performed, even if the length of the message is already congruent to 448 , modulo 512 (in which case 512 bits of padding are added).

Padding is performed as follows: a single " 1 " bit is appended to the message, and then enough zero bits are appended so that the length in bits of the padded message becomes congruent to 448 , modulo 512 . (This padding operation is invertible, so that different inputs yield different outputs-this would not be true if we merely padded with 0 's.)

\section{Step 2. Append length}

A 64-bit representation of $b$ (the length of the message before the padding bits were added) is appended to the result of the previous step. These bits are appended as two 32-bit words and appended low-order word first in accordance with the previous conventions. In the unlikely event that $b$ is greater than $2^{64}$, then only the low-order 64 bits of $b$ are used.

At this point the resulting message (after padding with bits and with $b$ ) has a length that is an exact multiple of 512 bits. Equivalently, this message has a length that is an exact multiple of 16 (32-bit) words. Let $M[0 \ldots N-1]$ denote the words of the resulting message, where $N$ is a multiple of 16 .

\section{Step 3. Initialize MD buffer}

A 4-word buffer $(A, B, C, D)$ is used to compute the message digest. Here each of $A, B, C, D$ is a 32 -bit register. These registers are initialized to the following values (in hexadecimal, low-order bytes first):

$\begin{array}{lllll}\text { word } A: & 01 & 23 & 45 & 67 \\ \text { word } B: & 89 & \text { ab } & \text { cd } & \text { ef } \\ \text { word } C: & \text { fe } & \text { dc } & \text { ba } & 98 \\ \text { word } D: & 76 & 54 & 32 & 10\end{array}$

\section{Step 4. Process message in 16-word blocks}

We first define three auxiliary functions that each take as input three 32-bit words and produce as output one 32 -bit word.

$$
\begin{aligned}
& f(X, Y, Z)=X Y \vee(\neg X) Z \\
& g(X, Y, Z)=X Y \vee X Z \vee Y Z \\
& h(X, Y, Z)=X \oplus Y \oplus Z
\end{aligned}
$$

In each bit position $f$ acts as a conditional: if $x$ then $y$ else $z$. In each bit position $g$ acts as a majority function: if at least two of $x, y, z$ are one, then $g$ has a one in that position. The function $h$ is the bit-wise xor or parity function. It is interesting 
to note that if the bits of $X, Y$, and $Z$ are independent and unbiased, the each bit of $f(X, Y, Z)$ is independent and unbiased, and similarly for $g(X, Y, Z)$ and $h(X, Y, Z)$.

MD4 utilizes two "magic constants" in rounds two and three. The round two constant is $\sqrt{2}$ and the round 3 constant is $\sqrt{3}$. (See Knuth [6, page 660].) Here are their values in octal and hex (with high-order digits given first).

\section{Octal Hex}

Round 2 constant $(\sqrt{2})$ : $013240474631 \quad 5$ A827999

Round 3 constant $(\sqrt{3})$ : 015666365641 6ED9EBA1

Do the following:

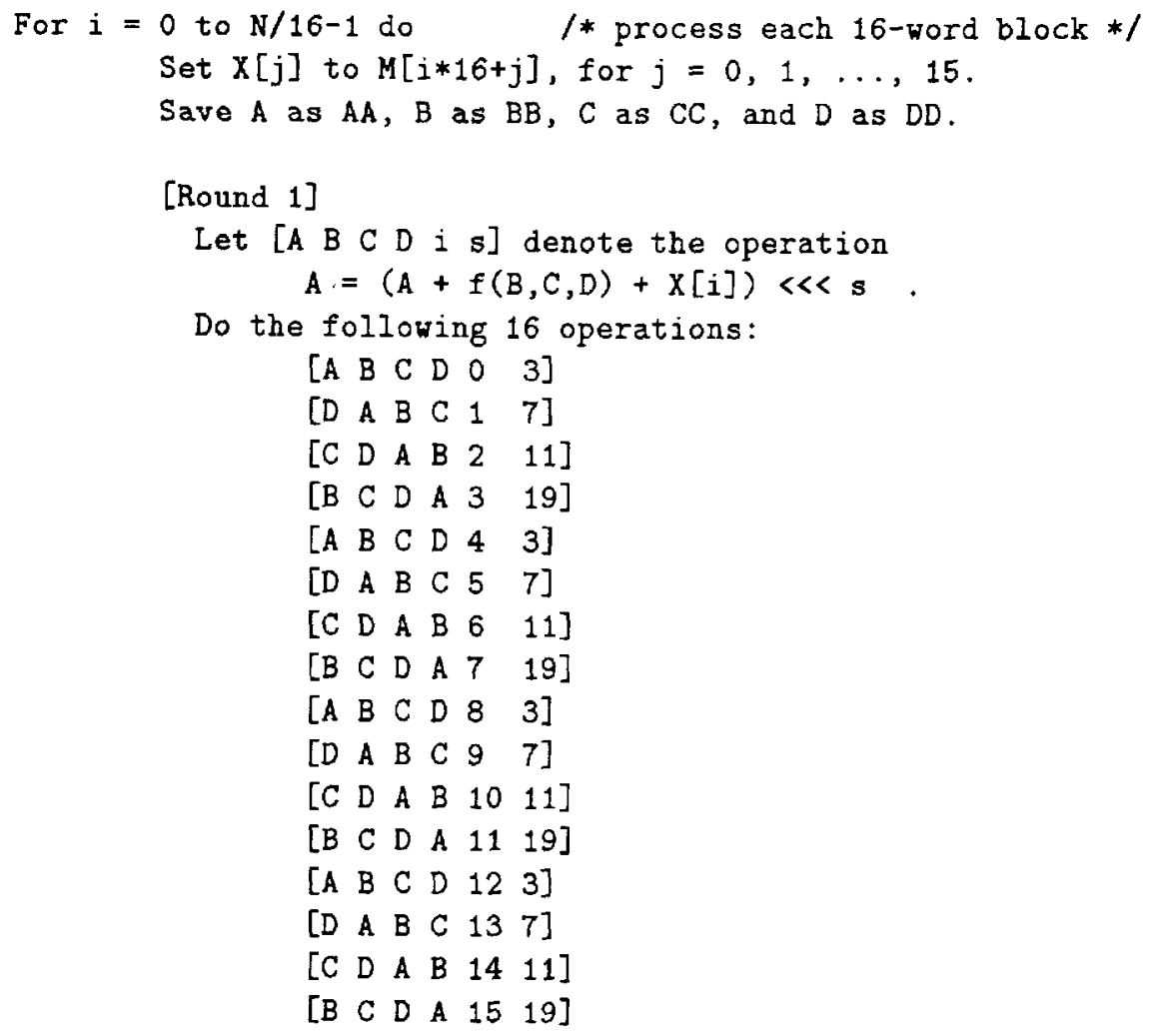



[D A
[C D $\left.\begin{array}{lllll} & A & B & 9 & 9\end{array}\right]$
$\left[\begin{array}{llllll}B & C & D & A & 13 & 13\end{array}\right]$
$\left[\begin{array}{llllll}A & B & C & D & 2 & 3\end{array}\right]$
[D A
[C D $\left.\begin{array}{lllll}\text { A } & B & 10 & 9\end{array}\right]$
$\left[\begin{array}{llllll}B & C & D & A & 14 & 13\end{array}\right]$
$\left[\begin{array}{llllll}A & B & C & D & 3 & 3\end{array}\right]$
$\left[\begin{array}{llllll}D & A & B & C & 7 & 5\end{array}\right]$
[C $D$ D A $\quad B \quad 11$ 9]
$\left[\begin{array}{llllll}B & C & D & A & 15 & 13\end{array}\right]$

\section{[Round 3]}

Let $[A B C D$ C $A=(A+h(B, C, D)+x[i]+6$ ED9EBA1 $) \ll s$. Do the following 16 operations:
$\left[\begin{array}{llllll}A & B & C & D & 0 & 3\end{array}\right]$
[D A
[C D $\left.\begin{array}{lllll} & \mathrm{B} & 4 & 11\end{array}\right]$

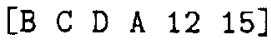
$\left[\begin{array}{llllll}A & B & C & D & 2 & 3\end{array}\right]$
[D A $\quad$ B $C$ C 10 9]
[C $\left.\begin{array}{llllll} & D & A & B & 6 & 11\end{array}\right]$
$\left[\begin{array}{lllllll}B & C & D & A & 14 & 15\end{array}\right]$
$\left[\begin{array}{llllll}A & B & C & D & 1 & 3\end{array}\right]$
[D A B
[C D A A B 5 (11]
$\left[\begin{array}{llllll}B & C & D & A & 13 & 15\end{array}\right]$
$\left[\begin{array}{llllll}A & B & C & D & 3 & 3\end{array}\right]$
[D A $\quad B \quad C \quad 11$ 9]
[C $\left.\begin{array}{lllll}\mathrm{D} & \mathrm{A} & \mathrm{B} & 7 & 11\end{array}\right]$
[B $C$ C $\left.\begin{array}{lllll} & \mathrm{A} & \mathrm{A} & 15 & 15\end{array}\right]$

Then perform the following additions:

$$
\begin{aligned}
& A=A+A A \\
& B=B+B B \\
& C=C+C C \\
& D=D+D D
\end{aligned}
$$

(That is, each of the four registers is incremented by the value it had before this block was started.)

end $/ *$ of loop on $i * /$ 


\section{Step 5. Output}

The message digest produced as output is $A, B, C, D$. That is, we begin with the low-order byte of $A$, and end with the high-order byte of $D$.

This completes the description of MD4.

\section{Extensions}

If more than 128 bits of output are required, then the following procedure is recommended to obtain a 256-bit output. No provision is made for obtaining more than 256 bits.

Two copies of MD4 are run in parallel over the input. The first copy is standard as described above. The second copy is modified as follows.

The initial state of the second copy is:

$\begin{array}{lllll}\text { word } A: & 00 & 11 & 22 & 33 \\ \text { word } B: & 44 & 55 & 66 & 77 \\ \text { word } C: & 88 & 99 & \text { aa } & \text { bb } \\ \text { word } D: & \text { cc } & \text { dd } & \text { ee } & \text { ff }\end{array}$

The magic constants in rounds 2 and 3 for the second copy of MD4 are changed from $\sqrt{2}$ and $\sqrt{3}$ to $\sqrt[3]{2}$ and $\sqrt[3]{3}$ :

Octal Hex

Round 2 constant $(\sqrt[3]{2}): \quad 012050505746 \quad 50$ a28be 6

Round 3 constant $(\sqrt[3]{3})$ : $013423350444 \quad 5 c 4$ dd124

Finally, after every 16-word block is processed (including the last block), the values of the $A$ registers in the two copies are exchanged.

The final message digest is obtaining by appending the result of the second copy of MD4 to the end of the result of the first copy of MD4.

\section{Implementation}

The MD4 algorithm has been implemented in $C$ and run on many different workstations. Here is a sampling of the running times achieved. (These running times include all processing, including byte-reversing the input words, but does not include file I/O time.) Assembly language implementations would of course be even faster.

$\begin{array}{lc}\text { Processor } & \text { Speed (Bytes/second) } \\ \text { SUN Sparc station } & 1,450,000 \\ \text { DEC MicroVax II } & 70,000 \\ 20 \mathrm{MHz} 80286 & 32,000\end{array}$


A reference implementation of MD4 in C is available as file md4. doc by anonymous ftp from theory.lcs.mit.edu and rsa.com, or from RSA Data Security, 10 Twin Dolphin Drive, Redwood City, California 94065, phone: 1-800-PUBLIKEY.

If you implement MD4 based on the description in this paper rather than the reference implementation, you may wish to check the following input-output values for MD4.

$\begin{array}{cc}\text { Input } & \text { Output } \\ \text { "" } & \text { 31d6cfe0d16ae931b73c59d7e0c089c0 } \\ \text { "a" } & \text { bde52cb31de33e46245e05fbdbd6fb24 } \\ \text { "abc" } & \text { a448017aaf21d8525fc10ae87aa6729d } \\ \text { "message digest" } & \text { d9130a8164549fe818874806e1c7014b } \\ \text { "abcdefghijklmnopqrstuvwxyz" } & \text { d79e1c308aa5bbcdeea8ed63df412da9 }\end{array}$

\section{Summary}

The MD4 message digest algorithm is simple to implement, and provides a "fingerprint" or message digest of a message of arbitrary length.

It is conjectured that the difficulty of coming up with two messages having the same message digest is on the order of $2^{64}$ operations, and that the difficulty of coming up with any message having a given message digest is on the order of $2^{128}$ operations. The MD4 algorithm has been carefully scrutinized for weaknesses. It is, however, a relatively new algorithm and further security analysis is of course justified, as is the case with any new proposal of this sort. ${ }^{1}$ The level of security provided by MD4 should be sufficient for implementing very high security hybrid digital signature schemes based on MD4 and the RSA public-key cryptosystem.

\section{Acknowledgments}

I'd like to thank Don Coppersmith, Burt Kaliski, Ralph Merkle, and Noam Nisan for numerous helpful comments and suggestions.

\section{References}

[1] Ivan Bjerre Damgård. A design principle for hash functions. In G. Brassard, editor, Proceedings CRYPTO 89, pages 416-427. Springer, 1990. Lecture Notes in Computer Science No. 435.

[2] D. W. Davies and W. L. Price. The application of digital signatures based on public-key cryptosystems. In Proc. Fifth Intl. Computer Communications Conference, pages 525-530, October 1980.

\footnotetext{
${ }^{1}$ Ralph Merkle has recently shown how to find two messages that collide in a modified form of MD4 that omits the third round. This technique does not seem to generalize to handle the full MD4 method, or even one in which the first or second round is omitted instead of the third.
} 
[3] W. Diffie and M. E. Hellman. New directions in cryptography. IEEE Trans. Inform. Theory, IT-22:644-654, November 1976.

[4] A. Evans, W. Kantrowitz, and E. Weiss. A user authentication scheme not requiring secrecy in the computer. CACM, 17:437-442, August 1974.

[5] Russell Impagliazzo, Leonid A. Levin, and Michael Luby. Pseudo-random generation from one-way functions. In Proc. 21th ACM Symposium on Theory of Computing, pages 12-24, Seattle, 1989. ACM.

[6] Donald E. Knuth. Seminumerical Algorithms, volume 2 of The Art of Computer Programming. Addison-Wesley, 1969. Second edition, 1981.

[7] Ralph C. Merkle. One way hash functions and DES. In G. Brassard, editor, Proceedings CRYPTO 89, pages 428-446. Springer, 1990. Lecture Notes in Computer Science No. 435 .

[8] M. Naor and M. Yung. Universal one-way hash functions and their cryptographic applications. In Proc. 21th ACM Symposium on Theory of Computing, pages 3343, Seattle, 1989. ACM.

[9] John Rompel. One-way functions are necessary and sufficient for secure signatures. In Proc. 22nd ACM Symposium on Theory of Computing, pages 387-394, Baltimore, Maryland, 1990. ACM.

[10] M. V. Wilkes. Time-sharing computer systems. Elsevier, 1975. Third edition. 